\title{
Solar radiation prediction based on ICA and HGAPSO for Kuhin City, Iran
}

\author{
Alibakhsh Kasaeian, Mehdi Mehrpooya, Mahsa Aghaie \\ AND MOHAMmad Hossein Ahmadi ${ }^{\mathrm{a}}$ \\ Department of Renewable Energies and Environmental, Faculty of New Science and Technologies, University of Tehran, \\ Tehran, Iran
}

Received 22 June 2015, Accepted 22 October 2015

\begin{abstract}
In this study, sun radiation prediction has been carried out by utilization of ICA and HGAPSO algorithms, based on some climatic parameters such as air temperature, relative humidity and wind speed. Air temperature, relative humidity and wind speed data have been measured by accurate measuring tools in Kuhin city. The application of these data networks to predict solar radiation in a similar situation have been taught to the system. The networks precision is shown by their correlation coefficient $\left(R^{2}\right)$ and mean square error (MSE). Due to the different climatic conditions, solar radiation is very difficult to be predicted. Thus, the trained networks have deviations; hence the networks with $R^{2}$ equal to 0.7 and above were acceptable. At the end, the solar radiation prediction was carried out for some the days of two years.
\end{abstract}

Key words: Solar radiation / correlation coefficient / mean square error / prediction

\section{Introduction}

Cleanliness, being cheap and lack of $\mathrm{CO}_{2}$ emissions have made solar energy as one of the foremost renewable energies. With the development of photovoltaic (PV) systems, large scale grid-connected PV power plants have been spread all over the world in recent years [1]. The integration of a large number of embedded PV generators will have far reaching consequences not only on the national transmission and generation systems, but also on the distribution networks. PV will be a very important generation source for the micro grid (MG). The tendency of countries to reduce the global carbon dioxide emissions increased the use of photovoltaic. Because of the variations in solar irradiation and weather conditions such as temperature and humidity, one cannot determine the precised amount of power production of a PV plant. In these conditions, any PV power producer will act as an uncontrollable and non-transmittable system in the utility network [2]. In order to cooperate with the power distribution schematization, the power operation and the demand side management, the generation forecast of gridconnected photovoltaic systems has an important role in

\footnotetext{
${ }^{a}$ Corresponding author:

mohammadhosein.ahmadi@gmail.com
}

scheduling investments in networks. The generation forecast, particularly the short term forecast, is valuable for grid operators in order to make decisions of grid operation whose power output depends on some external conditions like temperature, humidity and solar radiation. The atmospheric conditions such as irradiance and temperature are the factors momentous on the power of photovoltaic unit.

Accuracy of irradiance data will affect on the prediction results [3]. If the maximum power tracking algorithm is used, the PV output power will be highly related to the solar irradiance. In the moderate climates solar irradiance is defined by short time fluctuations, so in these places it is necessary to predict solar irradiance for attaining the power output. There are several developed stochastic models to predict the solar irradiance data such as autoregressive (AR), autoregressive moving average (ARMA), autoregressive integrated moving average (ARIMA) and Markov chain [4-7]. Stochastic models are based on the probability estimation, so it is difficult to predict solar irradiance accurately. These models need the basics of the precised definition of the problem domains as well as the identification of the mathematical functions. This is the reason of the big errors that were found in the results of stochastic models. In order to solve this problem, Artificial Intelligence (AI) techniques [8] have been applied 
for modeling and predicting of solar irradiance data. A new solar radiation forecast technique based on ICA and HGAPSO is proposed in this paper.

\section{Imperialist competitive algorithm (ICA)}

Imperialist competitive algorithm (ICA) is a computational method that is used to solve diverse types of optimization problems. ICA same as GA and PSO uses evolutionary system to solve the problem. ICA method is inspired of the human social evolution that begins with an initial population of $N$ countries which are generated randomly in different places. Based on the cost function, these countries are selected as the imperialist and the others are named as colonies. Colonies are divided between imperialists, based on the imperialists' power. The normalized cost of imperialist is defined as [9]:

$$
C_{n}=\max _{i}\left\{c_{i}\right\}-c_{n}
$$

where $c_{n}$ is the cost of $n_{t h}$ imperialist and $C_{n}$ is the normalized cost of the imperialists, $\max _{i}\left\{c_{i}\right\}$ is the highest cost among the imperialists. As a result, the power of each imperialist can be determined as [9]:

$$
p_{n}=\left|\frac{C_{n}}{\sum_{i=1}^{N_{\text {simp }}} C_{i}}\right|
$$

ICA method is based on the assimilation of the colonies by the imperialists and the competition between the imperialists to assimilate more colonies. The movement of colony to imperialist is shown by $x$ in Figure 1 and the functionality of $x$ is defined as followings:

$$
x \sim U(0, \theta, d)
$$

where $d$ is the distance between each colony and the imperialist, $\theta$ is a random number with uniform distribution between 1 and 2 , and $x$ is a random number with uniform distribution. Furthermore, a colony can achieve a better position during moving, so the situation of the colony and imperialist will change. The total cost of the imperialist can be determined by sum of the power of the imperialist country and percentage of the power of its colonies [9]:

$$
\begin{aligned}
T C_{n}=\operatorname{Cost} & \left(\text { imperialist }_{n}\right) \\
& + \text { gmean }_{\left\{\operatorname{Cost}\left(\text { colonies of empire }_{n}\right\}\right.}
\end{aligned}
$$

where $T C$ is the total cost of the $n_{t h}$ empire, and $n$ is a positive value ranging between 0 and 1 . Also $\xi$ is a positive number which is considered to be less than 1 . A little value for $\xi$ entails the total power of the empire to direct by the imperialist and with the increase value of $\xi$, the role of colonies in total power of an empire will increase.

The imperialist which cannot succeed the competition will lose its power and will collapse while the powerful imperialists remain. The results of this competition would

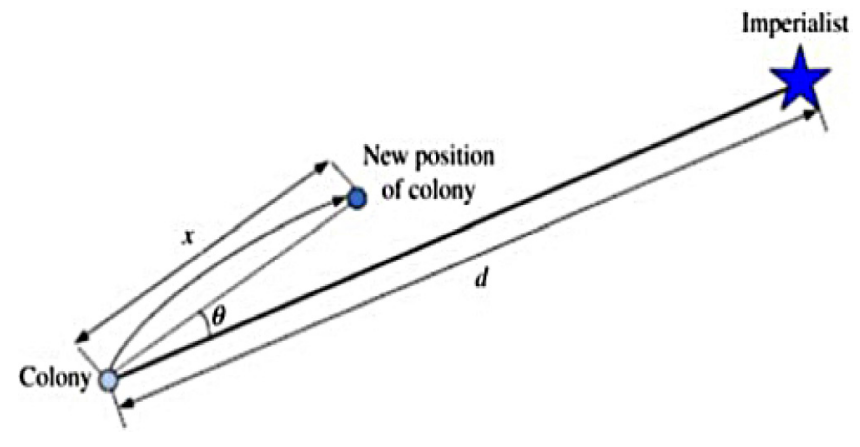

Fig. 1. Motion of colonies towards their imperialist [9].

increase the power of the succeeded imperialists and eliminate the powerless imperialists. In this state, the remained imperialist will reach the optimal point. Figure 2 shows the schematic of this method.

\section{Hybrid of genetic algorithm and particle swarm optimization (HGAPSO)}

A hybrid algorithm is a method which combines different algorithms to solve the same problem, either choosing one, or switching between them over the course of the algorithm. Because each algorithm has some desired features and some defects, a proper combination will benefit the favorable features of each one and finally the combined methods will give the best optimization. Nevertheless genetic algorithm has attained successful implementation within a wide range of problems [10-16]. HGAPSO is used for large-scale optimizations to contrast high computational efforts and costs; it would be great to combine genetic algorithm with particle swarm optimization in one algorithm. This is done in order to use the appropriate characteristics and search the abilities of both algorithms in predicting the desired parameters. In this study, the hybrid of genetic algorithm (GA) and particle swarm optimization (PSO) named HGAPSO, originally proposed by [14], is carried out to forecast the solar radiation. Figure 3 portrays the schematic representing the introduced hybrid of genetic algorithm with particle swarm optimization approach.

\section{Results and discussions}

ICA and HGAPSO algorithms are more optimized algorithms than neural networks and represent better models. In this study, for the purpose of training the network, three parameters including air temperature, relative humidity and wind speed are introduced as the inputs and sun radiation is the output. The air temperature, relative humidity and wind speed data have been measured by some accurate measuring tools in Kuhin city by our research team. Due to the difficulty of radiation prediction by air temperature, relative humidity, wind speed and 
A. Kasaeian et al.: Mechanics \& Industry 17, 509 (2016)

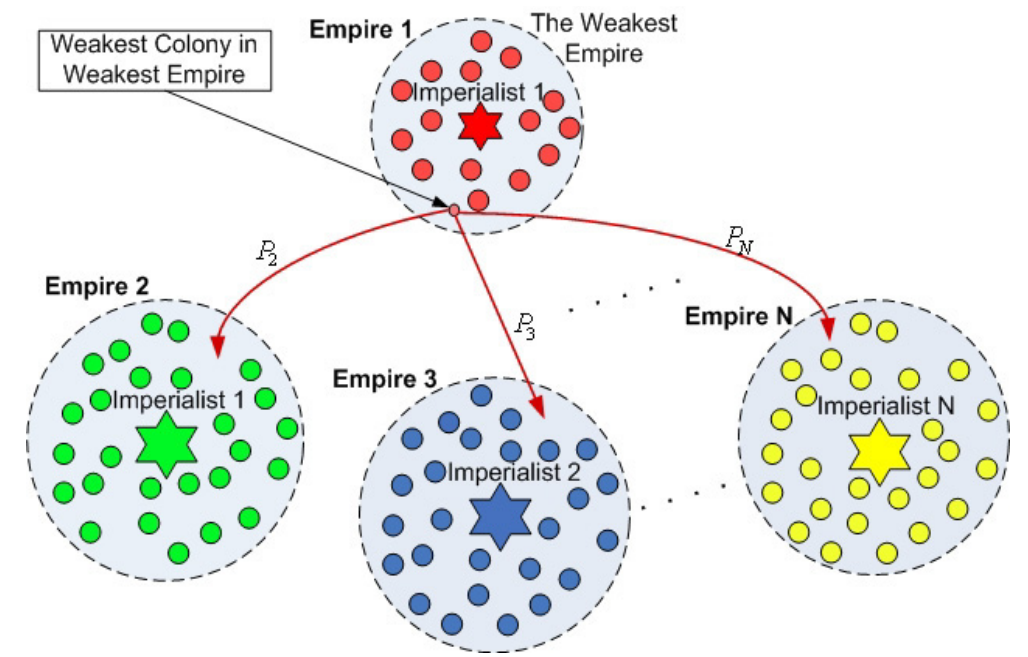

Fig. 2. Imperialistic competition [9].

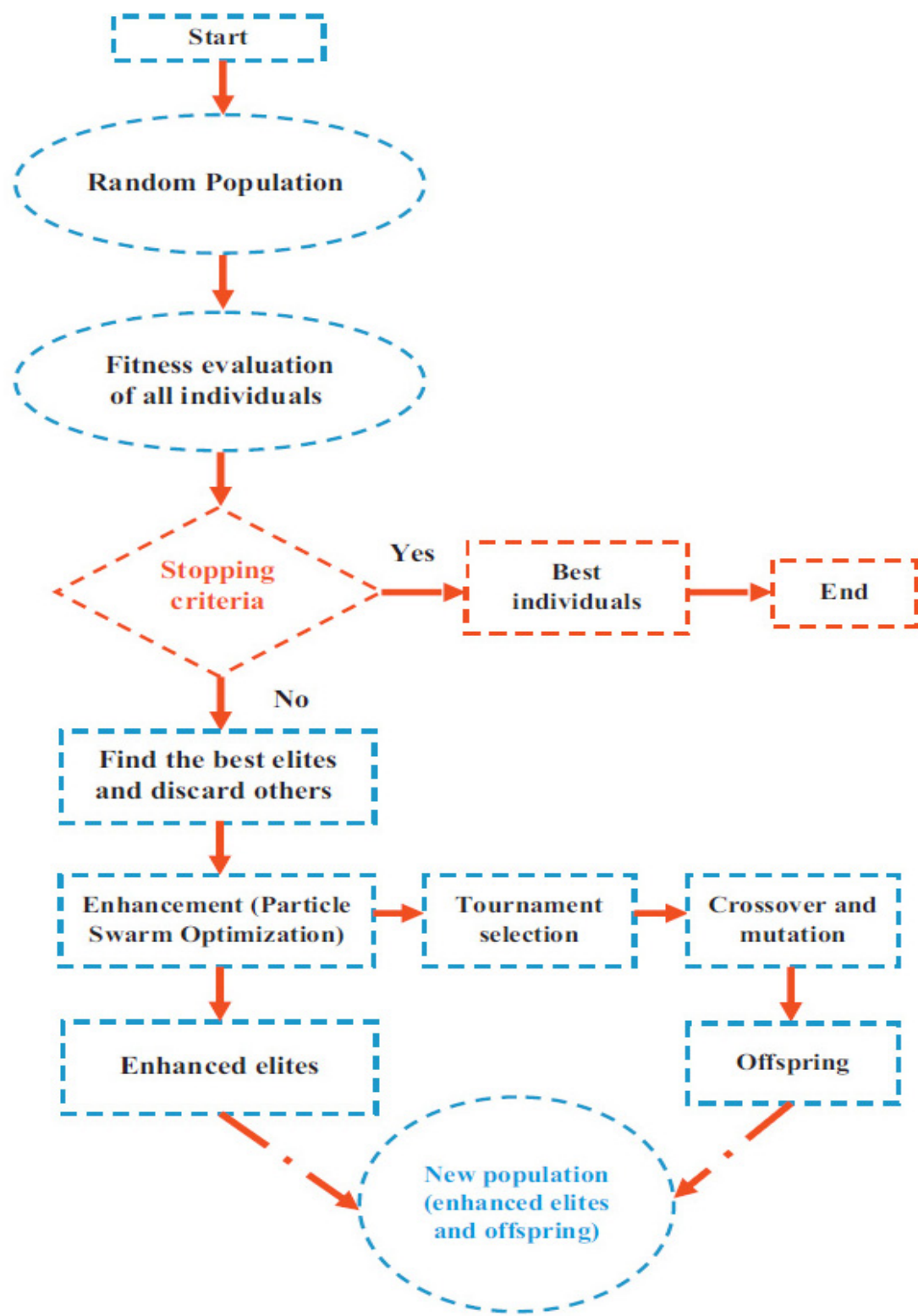

Fig. 3. Flowchart of the HGAPSO model $[10,14]$. 
A. Kasaeian et al.: Mechanics \& Industry 17, 509 (2016)

Table 1. Comparison between the performances of HGAPSO-ANN and ICA-ANN model for solar radiation.

\begin{tabular}{ccccc}
\hline \multirow{2}{*}{ Number of hidden neurons } & \multicolumn{2}{c}{ HGAPSO-ANN } & \multicolumn{2}{c}{ ICAANN } \\
\cline { 2 - 5 } & \multicolumn{2}{c}{ Training } & \multicolumn{2}{c}{ Training } \\
\cline { 2 - 5 } & MSE & $R^{2}$ & MSE & $R^{2}$ \\
\hline 3 & 0.28879 & 0.66189 & 0.27166 & 0.63807 \\
5 & 0.18308 & 0.55676 & 0.26634 & 0.57283 \\
7 & 0.20648 & 0.63147 & 0.24420 & 0.68536 \\
9 & 0.29512 & 0.67858 & 0.19613 & 0.68009 \\
11 & 0.16696 & 0.69333 & 0.23130 & 0.67925 \\
13 & 0.17577 & 0.70688 & 0.21580 & 0.65178 \\
17 & 0.20363 & 0.67681 & 0.21900 & 0.62783 \\
19 & 0.25464 & 0.71451 & 0.23644 & 0.69412 \\
21 & 0.19930 & 0.65952 & 0.20337 & 0.70530 \\
23 & 0.22365 & 0.67246 & 0.21813 & 0.69147 \\
& 0.18491 & 0.66468 & 0.27664 & 0.71885 \\
\hline
\end{tabular}
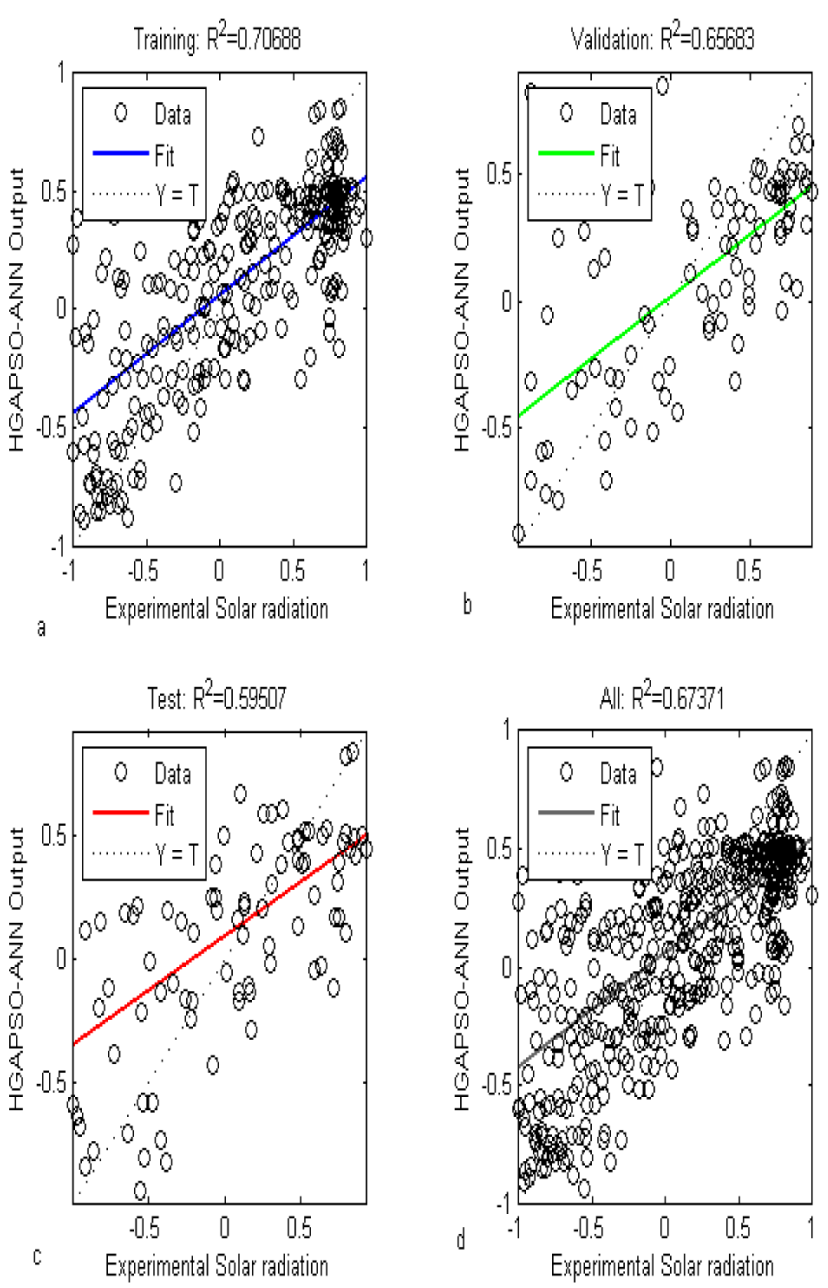

Fig. 4. $R^{2}$ for HGAPSO-ANN model for solar radiation.

other parameters, the trained networks with $R^{2}$ amounts of 0.7 and above are acceptable. The higher the coefficient of determination, the more the network is accurate.

As it is shown in Table 1, networks with different numbers of neurons in hidden layer have been trained with ICA and HGAPSO algorithms. In the networks which
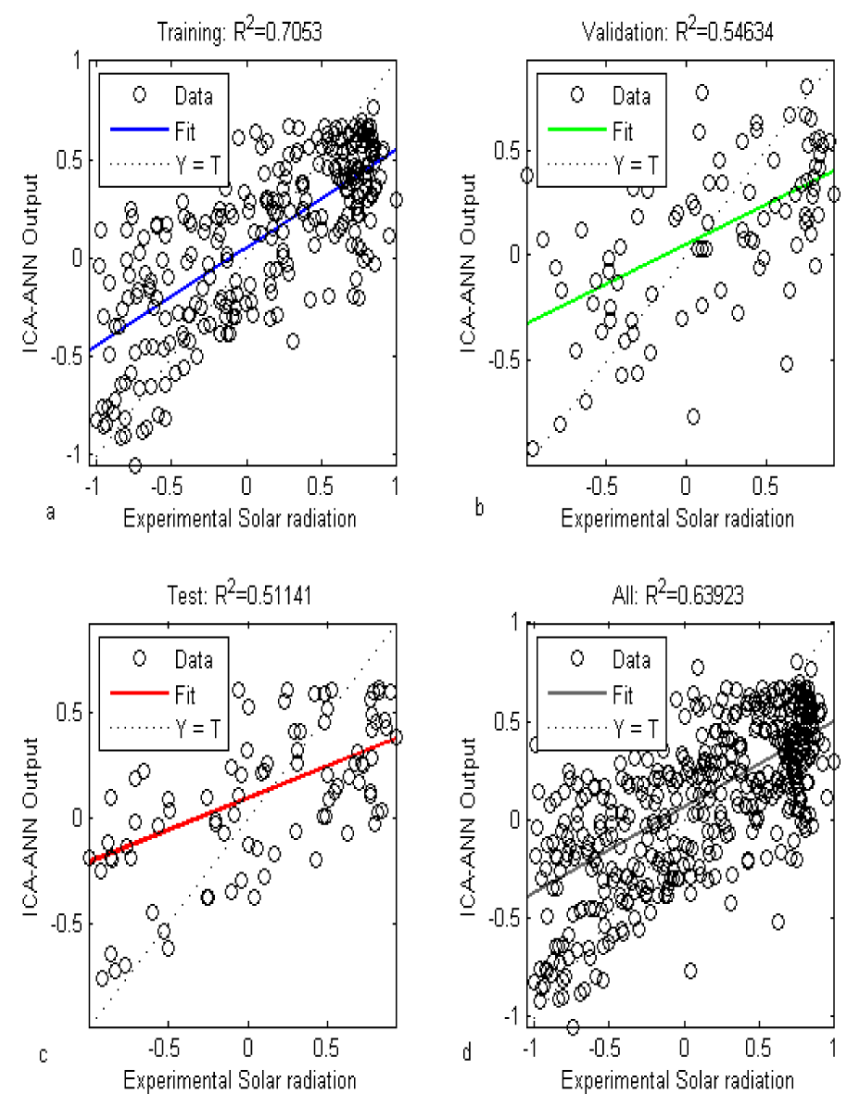

Fig. 5. $R^{2}$ for ICA-ANN model for solar radiation.

have been trained with ICA algorithm, the structure with 19 neurons and $R^{2}$ equal to 0.7053 (and MSE equal to 0.20337 ) is the best trained network. In the networks which have been trained with HGAPSO algorithm, the structure with 13 neurons and $R^{2}$ equal to 0.70668 (and MSE equal to 0.17577 ) is the best trained network.

Figure 4 shows the results of the trained network with HGAPSO algorithm with 13 neurons. Figure 4 a shows the trained network with $80 \%$ of the experimental data set. Figure $4 \mathrm{~b}$ presents the validation of the trained network with $10 \%$ of the experimental data set. Figure $4 \mathrm{c}$ shows 


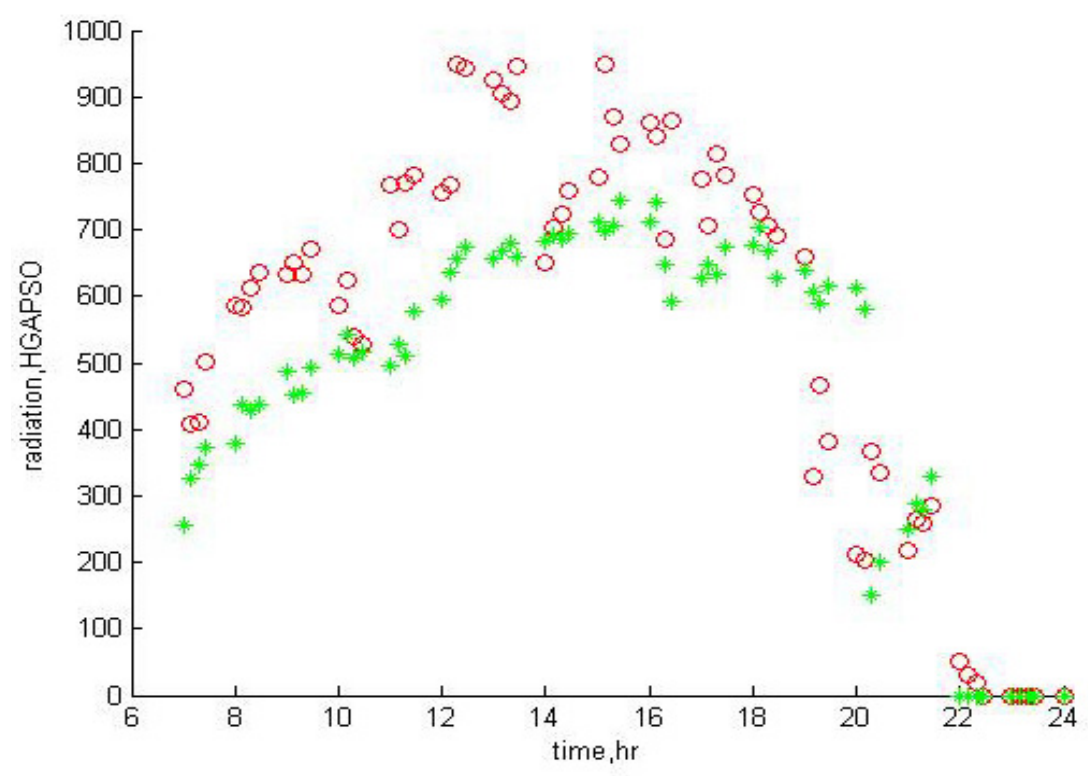

Fig. 6. Comparison between the experimental radiation and the HGAPSO model data .

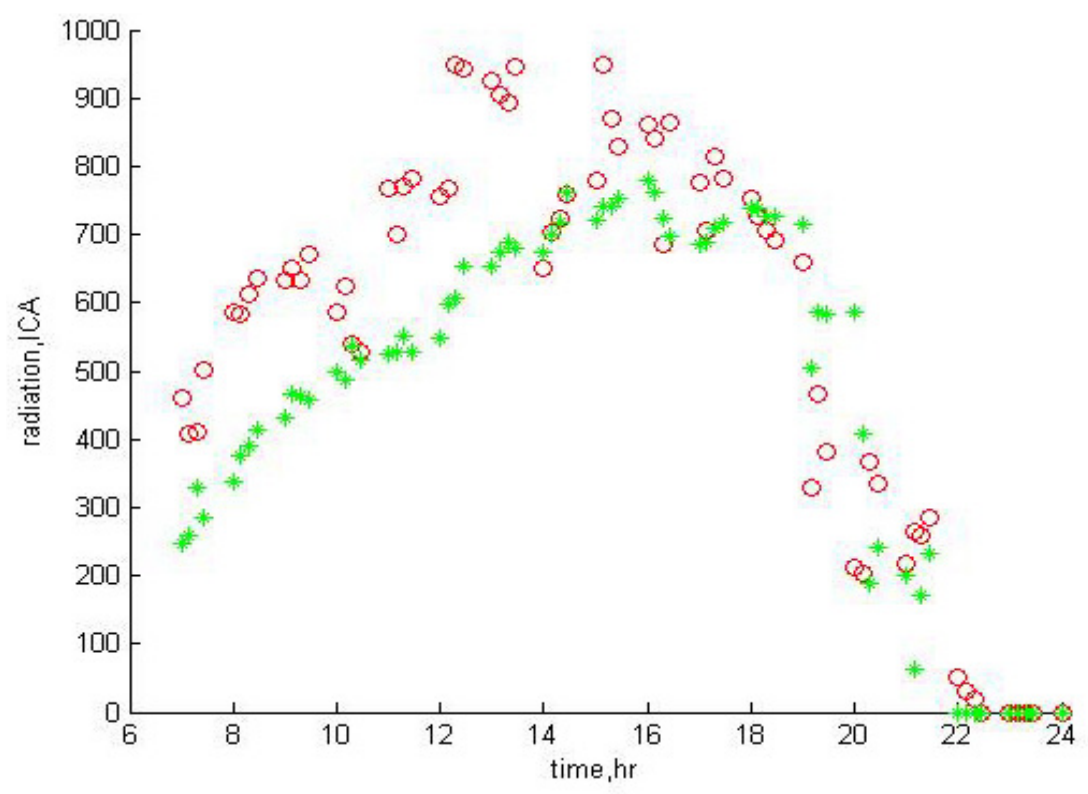

Fig. 7. Comparison between the experimental radiation and the ICA model data.

the trained network results with the rest unused $10 \%$ of the experimental data set. Figure $4 d$ depicts the trained network results with all of the experimental data.

Figure 5 demonstrates the results of the trained network with ICA algorithm with 19 neurons in which, 80\% of the experimental data were used to train the network, $10 \%$ for the validation and the last $10 \%$ for the test.

The comparison between the real data and the amounts data based on HGAPSO model is presented in Figure 6 . The comparison reveals a good conformity between the experimental data and the numerical results. The results are presented for one typical day and this model is capable to predict the radiation for all days of the year. For the purpose of validity check for ICA method, the obtained modeling results are compared with the experimental data in Figure 7. Again one can observe a good agreement with the experimental results

\section{Conclusion}

Study and prediction of solar radiation in Kuhin zone were carried out considering the weather conditions such as humidity, temperature and wind speed. The utilized data in this study are completely experimental which were measured with accurate measurement tools. For this forecast we used ICA and HGAPSO Meta-heuristic algorithms, which explain network using number of the neurons. The results of these algorithms are reported of which 
the most appropriate $R^{2}$ linear regression is 0.70668 . Finally with the same weather conditions the related solar radiations are predicted and show a good agreement with the experimental data.

\section{References}

[1] C. Tao, D. Shanxu, C. Changsong, Forecasting power output for grid-connected photovoltaic power system without using solar radiation measurement, In Power Electronics for Distributed Generation Systems (PEDG), 2nd IEEE International Symposium, 2010, pp. 773-777

[2] A. Woyte, V. Van Thong, R. Belmans, J. Nijs, Voltage fluctuations on distribution level introduced by photovoltaic systems, IEEE Trans. Energy Conversion 21 (2006) 202-209

[3] S. Cao, W. Weng, J. Chen, W. Liu, G. Yu, J. Cao, Forecast of Solar Irradiance Using Chaos Optimization Neural Networks, In Power and Energy Engineering Conference, APPEEC 2009, Asia-Pacific, pp. 1-4

[4] R. Aguiar, M. Collares-Pereira, TAG: A time-dependent, autoregressive, Gaussian model for generating synthetic hourly radiation, Solar Energy, 49 (1992) 167-174

[5] L.L. Mora-Lopez, M. Sidrach-de-Cardona, Multiplicative ARMA models to generate hourly series of global irradiation, Solar Energy 63 (1998) 283-291

[6] J.M. Santos, J.M. Pinazo, J. Canada, Methodology for generating daily clearness index values $\mathrm{Kt}$ starting from the monthly average daily value Kt. Determining the daily sequence using stochastic models, Renew. Energy 28 (2003) 1523-1544

[7] A. Maafi, A. Adane, A two-state Markovian model of global irradiation suitable for photovoltaic conversion, Solar Wind Technology 6 (1989) 247-252
[8] F.C. Morabito, M. Versaci, Fuzzy neural identification and forecasting techniques to process experimental urban air pollution data, Neural Networks 16 (2003) 493-506

[9] E. Atashpaz-Gargari, C. Lucas, Imperialist competitive algorithm: an algorithm for optimization inspired by imperialistic competition, In Evolutionary Computation, CEC 2007, IEEE Congress, 2007, pp. 4661-4667

[10] M.H Ahmadi, S. Sorouri Ghare Aghaj, A. Nazeri, Prediction of power in solar stirling heat engine by using neural network based on hybrid genetic algorithm and particle swarm optimization. Neural Computing and Application 22 (2013) 1141-1150

[11] X. Qu, J. Feng, W. Sun, Parallel genetic algorithm model based on AHP and neural networks for enterprise comprehensive business, IEEE Intelligent Conference on International Information Hiding and Multimedia Signal Processing, IIHMSP'08, pp. 897-900

[12] J.H. Doveton, S.E. Prensky, Geological applications of wire line logs: a synopsis of developments and trends, The Log Analyst 33 (1992) 286-303

[13] R. Reed, Pruning algorithms-a survey, IEEE Trans. Neural Networks, 4 (1993) 740-747

[14] M.H Ahmadi, M.A. Ahmadib, S.A. Sadatsakkakc, M. Feidtd, Connectionist intelligent model estimates output power and torque of stirling engine, Renewable and Sustainable Energy Reviews 50 (2015) 871-883

[15] M.H Ahmadi, M.A. Ahmadi, M. Mehrpooya, M.A. Rosen, Using GMDH Neural Networks to Model the Power and Torque of a Striling Engine, Sustainability 7 (2015) 2243-2255

[16] S.M. Pourkiaei, M.H. Ahmadi, and S.M. Hasheminejad, Modeling and experimental verification of a $25 \mathrm{~W}$ fabricated PEM fuel cell by parametric and GMDH-type neural network, Mechanic \& Industry 17 (2016) 105 